\title{
DERIVATIONS ON ALMOST COMPLEX MANIFOLDS
}

\author{
WEI XIA
}

\begin{abstract}
In this short note, we propose an unified method to derive formulas for derivations conjugated by exponential functions on an almost complex manifold.
\end{abstract}

\section{Introduction}

Our main result of this note is the following

Theorem 1.1. Let $(M, J)$ be an almost complex manifold, $E$ be a smooth vector bundle on $M$, and $\nabla$ be a linear connection on $E$ with the decomposition according to bidegrees : $\nabla=\nabla^{1,0}+\nabla^{0,1}-i_{\theta}-i_{\bar{\theta}}$, where $\theta \in A^{2,0}\left(M, T^{0,1}\right)$ is the torsion form of $J$. For any vector form $K \in A^{l+1}(M, T M), l \geq 0$, denote the interior derivative by $i_{K}$ and the Lie derivatives by $\mathcal{L}_{K}:=\left[i_{K}, \nabla\right], \mathcal{L}_{K}^{1,0}:=\left[i_{K}, \nabla^{1,0}\right], \mathcal{L}_{K}^{0,1}:=\left[i_{K}, \nabla^{0,1}\right]$, respectively. For any $\phi \in A^{0,1}\left(M, T^{1,0}\right)$ and $\psi \in A^{0,1}\left(M, T^{1,0}\right)$, set $e^{i_{\phi}}:=\sum_{k=0}^{\infty} \frac{i_{\phi}^{k}}{k !}$ and define $e^{i \bar{\phi}}, e^{i_{\psi}}, e^{i \bar{\psi}}$ in a similar way. Then we have

(1) $e^{-i_{\phi}} \nabla e^{i_{\phi}}=\nabla-\mathcal{L}_{\phi}-\frac{1}{2} i_{[\phi, \phi]}-\frac{1}{3 !} i_{[[\phi, \phi], \phi]^{\wedge}} ;$

(2) $e^{-i_{\phi}} \nabla^{1,0} e^{i_{\phi}}=\nabla^{1,0}-\mathcal{L}_{\phi}^{1,0}-\frac{1}{2} i_{[\phi, \phi]} A^{0,2}{\left(T^{1,0}\right)}$, $e^{-i_{\phi}} \nabla^{0,1} e^{i_{\phi}}=\nabla^{0,1}-\mathcal{L}_{\phi}^{0,1}$

(3) $e^{-i_{\phi}} i_{\theta} e^{i_{\phi}}=i_{\theta}+i_{[\theta, \phi]^{\wedge}}+\frac{1}{2} i_{[\theta, \phi]^{\wedge(2)}}+\frac{1}{3 !} i_{[\theta, \phi]^{\wedge(3)}}$, $e^{-i_{\phi}} i_{\bar{\theta}} e^{i_{\phi}}=i_{\bar{\theta}}$

(4) $e^{-i \bar{\psi}} i_{\phi} e^{i \bar{\psi}}=i_{\phi+[\phi, \bar{\psi}]^{\wedge}+[\phi, \bar{\psi}]^{\wedge(2)}}$,

$e^{-i \bar{\psi}} i_{[\phi, \phi]} e^{i \bar{\psi}}=i_{\sum_{j=0}^{j=3} \frac{1}{j !}[[\phi, \phi], \bar{\psi}]^{\wedge(j)}} ;$

(5) $e^{-i \bar{\psi}} \mathcal{L}_{\phi} e^{i \bar{\psi}}=\mathcal{L}_{\phi-i} \bar{\psi}_{\bar{\psi}}+i_{\sum_{j=0}^{j=2} \frac{1}{(j+1) !}[[\phi, \bar{\psi}], \bar{\psi}]^{\wedge(j)}}-i_{\sum_{j=0}^{j=2} \frac{1}{(j+2) !}[[i \bar{\psi} \phi, \bar{\psi}], \bar{\psi}]^{\wedge(2)}} ;$

(6) $e^{-i \bar{\psi}} e^{-i_{\phi}} \nabla e^{i_{\phi}} e^{i \bar{\psi}}=\nabla-\mathcal{L}_{\bar{\psi}}-\frac{1}{2} i_{[\bar{\psi}, \bar{\psi}]}-\frac{1}{3 !} i_{[[\bar{\psi}, \bar{\psi}], \bar{\psi}]^{\wedge}}-\mathcal{L}_{\phi-i \bar{\psi} \phi}-i_{\sum_{j=0}^{j=2} \frac{1}{(j+1) !}[[\phi, \bar{\psi}], \bar{\psi}]}{ }^{\wedge(j)}$ $+i_{\sum_{j=0}^{j=2} \frac{1}{(j+2) !}\left[\left[i_{\bar{\psi}} \phi, \bar{\psi}\right], \bar{\psi}\right]^{\wedge(2)}}-\frac{1}{2} i_{\sum_{j=0}^{j=3} \frac{1}{j !}[[\phi, \phi], \bar{\psi}]^{\wedge(j)}}-\frac{1}{3 !} i_{\sum_{j=0}^{j=3} \frac{1}{j !}\left[[[\phi, \phi], \phi]^{\wedge}, \bar{\psi}\right]^{\wedge(j)}}$.

NOTE : The corresponding formulas in a previous version of this paper [Xia19] are not correct. We mistakenly take $\left[A^{0,1}\left(M, T^{1,0}\right), A^{0,1}\left(M, T^{1,0}\right)\right] \in A^{0,2}\left(M, T^{1,0}\right)$ as granted which does not hold for general almost complex manifolds, see (2.3). The 
correct form of formulas (1), (2), (3) are first obtained by Fu-Liu in [FL19]. The main contribution of this note is thus to provide an alternative proof of these formulas.

If $J$ is integrable, then $\left[A^{0,1}\left(M, T^{1,0}\right), A^{0,1}\left(M, T^{1,0}\right)\right] \in A^{0,2}\left(M, T^{1,0}\right)$ holds and $(1),(2)$ is reduced to the extension formulas proved in [LRY15]. After reviewing some basic facts about derivations in Section 2, we prove our main result in Section 3.

\section{Derivations and BRaCket operations on a ReAl Manifold}

In this section we review some basic facts about derivations and refer the readers to chapter II of [KMS93] for more details. Let $M$ be a smooth manifold of dimension $n, A(M)=\bigoplus_{k=0}^{n} A^{k}(M)$ be its exterior algebra of differential forms. A (graded) derivation $D$ on $A(M)$ is a $\mathbb{R}$-linear map $D: A(M) \rightarrow A(M)$ with $D\left(A^{l}(M)\right) \subseteq$ $A^{l+k}(M)$ and $D(\xi \wedge \eta)=(D \xi) \wedge \eta+(-1)^{k l} \xi \wedge(D \eta)$ for $\xi \in A^{l}(M)$. The integer $k$ is called the degree of $D$. We denote by $D^{k}(M)$ the space of all derivations of degree $k$ on $A(M)$. For any $D_{1} \in D^{k_{1}}(M)$ and $D_{2} \in D^{k_{2}}(M)$, the graded commutator is defined by $\left[D_{1}, D_{2}\right]:=D_{1} D_{2}-(-1)^{k_{1} k_{2}} D_{2} D_{1}$. With respect to this bracket operation, the space of all derivations $D(M)=\bigoplus_{k} D^{k}(M)$ becomes a graded Lie algebra.

2.1. The interior derivative and Lie derivative. For a smooth vector field $X$ on $M$, we have the interior derivative $i_{X}$ which is a derivation of degree -1 . For a vector valued $(k+1)$-form $K \in A^{k+1}(M, T M)$, we can associate a derivation $i_{K}$ of degree $k$ by setting $i_{K} \varphi:=\xi \wedge\left(i_{X} \varphi\right)$, if $K=\xi \otimes X$ for a $(k+1)$-form $\xi$ and a vector field $X$, where $\varphi \in A(M)$. The exterior derivative $d$ is a derivation of degree 1 . The Lie derivative $\mathcal{L}_{X}:=\left[i_{X}, d\right]=i_{X} d+d i_{X}$ is a derivation of degree 0. Similarly, the Lie derivative $\mathcal{L}_{K}:=\left[i_{K}, d\right]=i_{K} d-(-1)^{k} d i_{K}$, where $K \in A^{k+1}(M, T M)$, is a derivation of degree $k+1$. In fact, for any $D \in D^{k}(M)$, there exist unique $K \in A^{k}(M, T M)$ and $L \in A^{k+1}(M, T M)$ such that

$$
D=\mathcal{L}_{K}+i_{L}
$$

and $L=0$ if and only if $[D, d]=0, K=0$ if and only if $D$ is algebraic.

2.2. Algebraic derivation and Nijenhuis-Richardson bracket. A derivation $D$ on $A(M)$ is called algebraic if $D f=0, \forall f \in A^{0}(M)$. Every algebraic derivation of degree $k$ on $A(M)$ is of the form $i_{K}$ for some unique $K \in A^{k+1}(M, T M)$. For any two algebraic derivation $i_{K} \in D^{k}(M)$ and $i_{L} \in D^{l}(M),\left[i_{K}, i_{L}\right]$ is again an algebraic derivation, hence $\left[i_{K}, i_{L}\right]=i_{[K, L]^{\wedge}}$ for some unique $[K, L]^{\wedge} \in A^{k+l+1}(M, T M)$. We have

$$
\left[i_{K}, i_{L}\right]=i_{[K, L]^{\wedge}}=i_{\left(i_{K} L-(-1)^{k l} i_{L} K\right)} .
$$

The operation $[\cdot, \cdot]^{\wedge}$ is called the Nijenhuis-Richardson bracket. $i_{K} L$ is defined as $i_{K} L:=\xi \wedge\left(i_{X} \eta\right) \otimes Y$ for $K=\xi \otimes X$ and $L=\eta \otimes Y$. 
2.3. The Frölicher-Nijenhuis bracket. For any $K \in A^{k}(M, T M), L \in A^{l}(M, T M)$, $\left[\mathcal{L}_{K}, \mathcal{L}_{L}\right]$ is a derivation of degree $k+l$ such that $\left[\left[\mathcal{L}_{K}, \mathcal{L}_{L}\right], d\right]=0$, hence $\left[\mathcal{L}_{K}, \mathcal{L}_{L}\right]=$ $\mathcal{L}_{[K, L]}$ for some unique $[K, L] \in A^{k+l}(M, T M)$. This operation $[\cdot, \cdot]$ is called the Frölicher-Nijenhuis bracket. For $k=l=0$, this bracket coincides with the Lie bracket of vector fields. On an almost complex manifold $(M, J)$, the Newlander-Nirenberg theorem says that $J$ is integrable if and only if $[J, J]=0$, where $J: T M \rightarrow T M$ is considered as a vector 1-form. On a complex manifold, the Frölicher-Nijenhuis bracket can be extended $\mathbb{C}$-linearly and is exactly the bracket operation appeared in Kodaira-Spencer's deformation theory [MK71]. Nevertheless, we should notice a vital difference between the integrable case and the general case: for $\phi \in A^{0,1}\left(M, T^{1,0}\right)$ and $\psi \in A^{0,1}\left(M, T^{1,0}\right),[\phi, \psi] \in A^{0,2}\left(M, T^{1,0}\right)$ does not always hold if $J$ is not integrable. In fact, we have ${ }^{1}$

$$
[\phi, \psi] \in A^{0,2}\left(M, T^{1,0}\right) \oplus A^{1,1}\left(M, T^{1,0}\right) \oplus A^{0,2}\left(M, T^{0,1}\right) .
$$

We denote by $[\phi, \psi]^{A^{0,2}\left(T^{1,0}\right)}$ the component of $[\phi, \psi]$ which lies in $A^{0,2}\left(M, T^{1,0}\right)$. Similarly, we write $[\phi, \psi]^{A^{1,1}\left(T^{1,0}\right)}$ and $[\phi, \psi]^{A^{0,2}\left(T^{0,1}\right)}$ for the other components.

2.4. A useful commutator relation. The following commutator relation will be useful for our purpose: for $K \in A^{k}(M, T M), L \in A^{l+1}(M, T M)$, we have

$$
\left[\mathcal{L}_{K}, i_{L}\right]=i_{[K, L]}-(-1)^{k l} \mathcal{L}_{i_{L} K}
$$

See [Mic86], [LR11] and [LRY15] for various forms and generalizations of this formula.

\section{DeRIVATIONS ON AN ALMOST COMPLEX MANIFOLD}

Now, let $(M, J)$ be an almost complex manifold of real dimension $2 n$, its complexified tangent bundle $T_{\mathbb{C}}(M)=T_{\mathbb{R}}(M) \otimes \mathbb{C}$ admits a decomposition $T_{\mathbb{C}}(M)=T^{1,0} \oplus T^{0,1}$. For each $k \leq 0$, the space of (complexified) $k$-forms $A_{\mathbb{C}}^{k}(M):=A^{k}(M) \otimes \mathbb{C}$ admits a decomposition $A^{k}(M)=\bigoplus_{p+q=k} A^{p, q}(M)$, where $A^{p, q}(M)=\wedge^{p} T^{* 1,0} \otimes \wedge^{q} T^{* 0,1}$. Hence $A_{\mathbb{C}}(M):=A(M) \otimes \mathbb{C}=A(E)$. In what follows, we omit the subscript "C্" and make the convention that differential forms are always complex valued unless otherwise stated. Replacing $\mathbb{R}$-linearity by $\mathbb{C}$-linearity, the notion of graded derivation on $A(M)$ is similarly defined. Since now $A(M)$ has a bigrading structure, we can make a refinement. A bigraded derivation of bidegree $(k, l)$ on $A(M)$ is a $\mathbb{C}$ linear map $D: A(M) \rightarrow A(M)$ with $D\left(A^{p, q}(M)\right) \subseteq A^{p+k, q+l}(M)$ and $D(\xi \wedge \eta)=$ $(D \xi) \wedge \eta+(-1)^{(k+l) m} \xi \wedge(D \eta)$ for $\xi \in A^{m}(M)$. By definition, A bigraded derivation of bidegree $(k, l)$ is necessarily a graded derivation of degree $k+l$. The space of all bigraded derivation of bidegree $(k, l)$ on $A(M)$ is denoted by $D^{k, l}(M)$, then $D^{k}(M)=\bigoplus_{p+q=k} D^{p, q}(M)$. Note that, by extending $\mathbb{C}$-linearly, all the constructions in previous section can be applied in the present situation.

\footnotetext{
${ }^{1}$ I owed this observation to Hai-Sheng Liu, see Lemma 3.3 of [FL19] and Theorem 8.7, (6) of [KMS93, pp. 70].
} 
Example 3.1 (c.f. chapter VIII of [Dem12]). The exterior derivative $d$ admit a decomposition into 4 bigraded derivations:

$$
d=\partial+\bar{\partial}-i_{\theta}-i_{\bar{\theta}}
$$

where $\partial:=\sum_{p, q} \Pi^{p+1, q} d \Pi^{p, q}$ with $\Pi^{p, q}$ being the projection $A(M) \rightarrow A^{p, q}(M)$ and $\bar{\partial}:=\sum_{p, q} \Pi^{p, q+1} d \Pi^{p, q}$. And $\theta \in A^{2,0}\left(M, T^{0,1}\right)$ is the torsion form of $J$ which is defined by $\theta(X, Y):=[X, Y]^{0,1}$ for $X, Y \in A^{0}\left(M, T^{1,0}\right)$, where $[X, Y]^{0,1}$ is the $(0,1)$ part of the vector field $[X, Y]$. We see that the bidegree of $\partial, \bar{\partial}, i_{\theta}, i_{\bar{\theta}}$ are $(1,0),(0,1),(2,-1),(-1,2)$ respectively.

Example 3.2. For $\phi \in A^{0, k}\left(M, T^{1,0}\right)$, define

$$
\mathcal{L}_{\phi}^{1,0}:=\left[i_{\phi}, \partial\right]=i_{\phi} \partial-\partial i_{\phi} \text { and } \mathcal{L}_{\phi}^{0,1}:=\left[i_{\phi}, \bar{\partial}\right]=i_{\phi} \bar{\partial}-\bar{\partial} i_{\phi},
$$

then $\mathcal{L}_{\phi}^{1,0} \in D^{0, k}(M)$ and $\mathcal{L}_{\phi}^{0,1} \in D^{-1, k+1}(M)$.

It is clear that if $D \in D^{p, q}(M)$ is algebraic, $D=i_{L}$ for some unique $L \in$ $A^{p+1, q}\left(M, T^{1,0}\right) \oplus A^{p, q+1}\left(M, T^{0,1}\right)$. We can also make the following refinement of (2.1):

Proposition 3.3. Let $D \in D^{p, q}(M)$, then we have

$$
D=\mathcal{L}_{K^{\prime p, q}}^{1,0}+\mathcal{L}_{K^{\prime \prime p, q}}^{0,1}+i_{L^{\prime p+1, q}}+i_{L^{\prime \prime} p, q+1},
$$

for some $K^{\prime p, q} \in A^{p, q}\left(M, T^{1,0}\right), K^{\prime \prime} p, q \in A^{p, q}\left(M, T^{0,1}\right)$ and $L^{\prime p, q} \in A^{p, q}\left(M, T^{1,0}\right)$, $L^{\prime \prime} p, q \in A^{p, q}\left(M, T^{0,1}\right)$.

Proof. Let $D \in D^{p, q}(M)$, then $D=\mathcal{L}_{K}+i_{L}$, for some unique $K \in A^{p+q}(M, T M)$ and $L \in A^{p+q+1}(M, T M)$. We can write

$$
D=\sum_{a+b=p+q}\left(\mathcal{L}_{K^{\prime} a, b}^{1,0}+\mathcal{L}_{K^{\prime \prime a} a b}^{1,0}+\mathcal{L}_{K^{\prime} a, b}^{0,1}+\mathcal{L}_{K^{\prime \prime a} a b}^{0,1}\right)+\sum_{a+b=p+q+1} i_{L^{\prime} a, b+L^{\prime \prime a}, b},
$$

where $\forall a, b, K^{\prime a, b} \in A^{a, b}\left(M, T^{1,0}\right), K^{\prime \prime} a, b \in A^{a, b}\left(M, T^{0,1}\right)$ and similarly $L^{\prime a, b} \in A^{a, b}\left(M, T^{1,0}\right)$, $L^{\prime \prime} a, b \in A^{a, b}\left(M, T^{0,1}\right)$. It follows from $D^{k}(M)=\bigoplus_{p+q=k} D^{p, q}(M)$ that

$$
D=\mathcal{L}_{K^{\prime} p, q}^{1,0}+\mathcal{L}_{K^{\prime \prime} p-1, q+1}^{1,0}+\mathcal{L}_{K^{\prime} p+1, q-1}^{0,1}+\mathcal{L}_{K^{\prime \prime} p, q}^{0,1}+i_{L^{\prime} p+1, q}+i_{L^{\prime \prime} p, q+1} .
$$

It is clear that $\mathcal{L}_{K^{\prime \prime} p-1, q+1}^{1,0}$ and $\mathcal{L}_{K^{\prime} p+1, q-1}^{0,1}$ are algebraic so that $\mathcal{L}_{K^{\prime \prime} p-1, q+1}^{1,0}=i_{R^{\prime \prime p, q+1}}$, $\mathcal{L}_{K^{\prime} p+1, q-1}^{0,1}=i_{R^{\prime} p+1, q}$ for some $R^{\prime \prime p, q+1} \in A^{p, q+1}\left(M, T^{0,1}\right)$ and $R^{\prime p+1, q} \in A^{p+1, q}\left(M, T^{1,0}\right)$.

Remark 3.4. It is important to notice that the uniqueness part of (2.1) is lost.

Let $\phi \in A^{0,1}\left(M, T^{1,0}\right)$, then $i_{\phi}$ is nilpotent : $\left(i_{\phi}\right)^{n+1} \xi=0, \forall \xi \in A(M)$, so that the operator

$$
e^{i_{\phi}}:=\sum_{k=0}^{\infty} \frac{i_{\phi}^{k}}{k !}: A(M) \longrightarrow A(M)
$$


is well-defined. Since $e^{i_{\phi}} e^{-i_{\phi}}=e^{-i_{\phi}} e^{i_{\phi}}=e^{0}$ is the identity operator, $e^{-i_{\phi}}$ is the inverse operator of $e^{i_{\phi}}$.

Definition 3.5. Let $R$ be an unitary associative algebra over $\mathbb{Q}$ (not necessarily commutative). For any $x, y \in R$, we say that $x$ is finitely commutable with $y$ if there is a positive integer $k$ such that

$$
\underbrace{[\cdots[}_{k \text { times }} x, \overbrace{y], y], \cdots, y]}^{k \text { times }}=0,
$$

where $[x, y]=x y-y x$ is the usual commutator. If $x$ is finitely commutable with $y$, the least integer $k$ such that (3.2) holds is called the commutable degree of $(x, y)$, and in this case we say $x$ is $k$-commutable with $y$. We will simply denote the $k$ times bracket in $(3.2)$ by $[x, y]^{(k)}$ and make the convention that $[x, y]^{(0)}:=x$.

3.1. A commutator lemma. The following lemma is perhaps well-known to experts, see [Got05, Lem. 2.7] and [Man05, pp.66, Exercise V.1.]. For the readers' convenience, we will present here two different proofs.

Lemma 3.6. Let $R$ be an unitary associative algebra over $\mathbb{Q}$ (not necessarily commutative), $y \in R$ be a nilpotent element. Assume $x \in R$ is $k$-commutable with $y$, i.e. $[x, y]^{(k)}=0$, then

$$
e^{-y} x e^{y}=\sum_{i=0}^{i=k-1} \frac{1}{i !}[x, y]^{(i)},
$$

where $e^{y}:=1+y+\frac{y^{2}}{2 !}+\cdots$ is the exponential function.

Proof. First, we set

$$
f(t):=e^{-t y} x e^{t y}
$$

where $t$ is a real variable. It can be proved inductively that

$$
\frac{d^{k} f}{d t^{k}}(0)=[x, y]^{(k)}
$$

Hence, we have

$$
e^{-y} x e^{y}=f(1)=\sum_{k} \frac{d^{k} f}{d t^{k}}(0) \frac{1}{k !}=\sum_{k} \frac{1}{k !}[x, y]^{(k)} .
$$


Proof. Assume $y^{l}=0$ for some positive integer $l$ and set $N=\max \{k, l\}$, then

$$
\begin{aligned}
x e^{y}= & x\left(1+y+\frac{y^{2}}{2 !}+\cdots\right) \\
= & x+x y\left(1+\frac{y}{2 !}+\frac{y^{2}}{3 !}+\cdots\right) \\
= & x+([x, y]+y x)\left(1+\frac{y}{2 !}+\frac{y^{2}}{3 !}+\cdots\right) \\
= & (1+y) x+[x, y]+([x, y]+y x)\left(\frac{y}{2 !}+\frac{y^{2}}{3 !}+\cdots\right) \\
= & (1+y) x+[x, y]+\left([x, y]^{(2)}+y[x, y]+y[x, y]+y^{2} x\right)\left(\frac{1}{2 !}+\frac{y}{3 !}+\cdots\right) \\
= & \sum_{i=0}^{i=2} \frac{y^{i}}{i !} x+(1+y)[x, y]+\frac{1}{2}[x, y]^{(2)}+\left([x, y]^{(2)}+2 y[x, y]+y^{2} x\right)\left(\frac{y}{3 !}+\cdots\right) \\
= & \sum_{i=0}^{i=3} \frac{y^{i}}{i !} x+\sum_{i=0}^{i=2} \frac{y^{i}}{i !}[x, y]+\frac{1}{2}(1+y)[x, y]^{(2)}+\frac{1}{3 !}[x, y]^{(3)} \\
+ & \left([x, y]^{(3)}+3 y[x, y]^{(2)}+3 y^{2}[x, y]+y^{3} x\right)\left(\frac{y}{4 !}+\cdots\right) \\
= & \sum_{i=0}^{i=4} \frac{y^{i}}{i !} x+\sum_{i=0}^{i=3} \frac{y^{i}}{i !}[x, y]+\frac{1}{2} \sum_{i=0}^{i=2} \frac{y^{i}}{i !}[x, y]^{(2)}+\frac{1}{3 !}(1+y)[x, y]^{(3)}+\frac{1}{4 !}[x, y]^{(4)} \\
+ & \left([x, y]^{(4)}+4 y[x, y]^{(3)}+6 y^{2}[x, y]^{(2)}+4 y^{3}[x, y]+y^{4} x\right)\left(\frac{y}{5 !}+\cdots\right) \\
= & \cdots=\sum_{i=0}^{i=2 N} \frac{y^{i}}{i !} x+\sum_{i=0}^{i=2 N-1} \frac{y^{i}}{i !}[x, y]+\cdots+\frac{1}{N !} \sum_{i=0}^{i=N} \frac{y^{i}}{i !}[x, y]^{(N)}+\cdots+\frac{1}{(2 N) !}[x, y]^{(2 N)} \\
+ & \sum_{i=0}^{i=2 N}(2 N) y^{i}[x, y]^{(2 N-i)}\left(\frac{y}{(2 N+1) !}+\cdots\right), \\
& =0 \\
& (2 N) \\
&
\end{aligned}
$$

where $\left(\begin{array}{c}2 N \\ i\end{array}\right)$ are the binomial coefficients. Now, by our assumption, we know that

$$
\sum_{i=0}^{i=2 N}\left(\begin{array}{c}
2 N \\
i
\end{array}\right) y^{i}[x, y]^{(2 N-i)}=0 \text { and } \sum_{i=0}^{i=2 N} \frac{y^{i}}{i !}=\sum_{i=0}^{i=2 N-1} \frac{y^{i}}{i !}=\cdots=\sum_{i=0}^{i=N} \frac{y^{i}}{i !}=e^{y},
$$

thus $x e^{y}=e^{y} \sum_{i=0}^{i=k-1} \frac{1}{i !}[x, y]^{(i)} \Rightarrow e^{-y} x e^{y}=\sum_{i=0}^{i=k-1} \frac{1}{i !}[x, y]^{(i)}$.

3.2. Derivations on the algebra of vector bundle valued forms. Let $E$ be a smooth vector bundle on the the almost complex manifold $(M, J)$ and $\nabla$ be a linear connection on $E$. The space of E-valued differential forms $A(E)$ can be decomposed as $A(E)=\bigoplus_{p, q} A^{p, q}(E)$. From the work of Michor [Mic86], we know that a similar theory as those described in Section 2 holds in this setting, in particular, formulas 
(2.2) and (2.4) are valid with the Lie derivative defined by $\mathcal{L}_{K}:=\left[i_{K}, \nabla\right]=i_{K} \nabla-$ $(-1)^{k} \nabla i_{K}$, where $K \in A^{k+1}(M, T M)$. See [Mic86, Th. 3.16] for the proof of (2.4). The space of all bigraded derivation of bidegree $(k, l)$ on $A(E)$ is denoted by $D^{k, l}(E)$, then $D^{k}(E)=\bigoplus_{p+q=k} D^{p, q}(E)$.

As in Example 3.1, the connection $\nabla$ admits a decomposition ${ }^{2}$ :

$$
\nabla=\nabla^{1,0}+\nabla^{0,1}-i_{\theta}-i_{\bar{\theta}}
$$

where $\nabla^{1,0}:=\sum_{p, q} \Pi^{p+1, q} \nabla \Pi^{p, q}$ with $\Pi^{p, q}$ being the projection $A(E) \rightarrow A^{p, q}(E)$ and $\nabla^{0,1}:=\sum_{p, q} \Pi^{p, q+1} \nabla \Pi^{p, q}$. Define $\mathcal{L}_{K}^{1,0}:=\left[i_{K}, \nabla^{1,0}\right]=i_{K} \nabla^{1,0}-\nabla^{1,0} i_{K}$ and $\mathcal{L}_{K}^{0,1}:=\left[i_{K}, \nabla^{0,1}\right]=i_{K} \nabla^{0,1}-\nabla^{0,1} i_{K}$ as usual. We make two observations. For $\phi \in A^{0, k}\left(M, T^{1,0}\right), \psi \in A^{0, l}\left(M, T^{1,0}\right)$, it follows easily form the definition that

$$
i_{\phi} i_{\psi}=(-1)^{(k+1)(l+1)} i_{\psi} i_{\phi},
$$

and since $i_{\psi} \phi=0$, by (2.4) we have

$$
\left[\mathcal{L}_{\phi}, i_{\psi}\right]=i_{[\phi, \psi]} \cdot
$$

Lemma 3.7. For $\phi \in A^{0,1}\left(M, T^{1,0}\right), \psi \in A^{0,1}\left(M, T^{1,0}\right)$, we have

1. $\left[\mathcal{L}_{\phi}^{1,0}, i_{\psi}\right]=i_{[\phi, \psi]]^{A^{0,2}\left(T^{1,0}\right)}} ;$

2. $\left[\mathcal{L}_{\phi}^{0,1}, i_{\psi}\right]=0$;

3. $-\left[[\phi, \theta]^{\wedge}, \psi\right]^{\wedge}=[\phi, \psi]^{A^{1,1}\left(T^{1,0}\right)}+[\phi, \psi]^{A^{0,2}\left(T^{0,1}\right)}$.

Proof. Since

$$
\mathcal{L}_{\phi}=\mathcal{L}_{\phi}^{1,0}+\mathcal{L}_{\phi}^{0,1}-i_{[\phi, \theta] \wedge} \in D^{0,1}(E) \oplus D^{-1,2}(E) \oplus D^{1,0}(E),
$$

where $[\phi, \theta]^{\wedge} \in A^{1,1}\left(M, T^{0,1}\right) \oplus A^{2,0}\left(M, T^{1,0}\right)$, we have

$$
\left[\mathcal{L}_{\phi}, i_{\psi}\right]=\left[\mathcal{L}^{1,0}, i_{\psi}\right]+\left[\mathcal{L}_{\phi}^{0,1}, i_{\psi}\right]-\left[i_{[\phi, \theta] \wedge}, i_{\psi}\right] \in D^{-1,2}(E) \oplus D^{-2,3}(E) \oplus D^{0,1}(E) .
$$

The conclusion then follows from (3.5) and (2.3).

As in (3.2), we use $[x, y]^{\wedge(k)}$ to denote the $k$-times Nijenhuis-Richardson bracket of $x$ with $y$, and $[x, y]^{\wedge(0)}:=x$.

Theorem 3.8. Let $\phi \in A^{0,1}\left(M, T^{1,0}\right)$ and $\psi \in A^{0,1}\left(M, T^{1,0}\right)$, then we have

\footnotetext{
${ }^{2}$ This can be checked easily. In fact, for $u \in A^{0}(M, E)$ this is clear; for $u \in A^{1}(M, E)$, write locally $u=\alpha_{i} \otimes s_{i}$, where $\alpha_{i}$ are 1-forms and $s_{i}$ is a local smooth frame, then we have $\nabla u=\nabla\left(\alpha_{i} \otimes s_{i}\right)=$ $d \alpha_{i} \otimes s_{i}-\alpha_{i} \otimes\left(\nabla^{1,0}+\nabla^{0,1}\right) s_{i}=\left(\nabla^{1,0}+\nabla^{0,1}-i_{\theta}-i_{\bar{\theta}}\right) u$.
} 
(1) $e^{-i_{\phi}} \nabla e^{i_{\phi}}=\nabla-\mathcal{L}_{\phi}-\frac{1}{2} i_{[\phi, \phi]}-\frac{1}{3 !} i_{[[\phi, \phi], \phi]^{\wedge}}$;

(2) $e^{-i_{\phi}} \nabla^{1,0} e^{i_{\phi}}=\nabla^{1,0}-\mathcal{L}_{\phi}^{1,0}-\frac{1}{2} i_{[\phi, \phi]} A^{A^{0,2}\left(T^{1,0}\right)}$, $e^{-i_{\phi}} \nabla^{0,1} e^{i_{\phi}}=\nabla^{0,1}-\mathcal{L}_{\phi}^{0,1}$

(3) $e^{-i_{\phi}} i_{\theta} e^{i_{\phi}}=i_{\theta}+i_{[\theta, \phi]^{\wedge}}+\frac{1}{2} i_{[\theta, \phi] \wedge(2)}+\frac{1}{3 !} i_{[\theta, \phi]^{\wedge(3)}}$,

$$
e^{-i_{\phi}} i_{\bar{\theta}} e^{i_{\phi}}=i_{\bar{\theta}}
$$

(4) $e^{-i_{\bar{\psi}}} i_{\phi} e^{i \bar{\psi}}=i_{\phi+[\phi, \bar{\psi}]^{\wedge}+[\phi, \bar{\psi}]^{\wedge(2)}}$,

$$
e^{-i \bar{\psi}} i_{[\phi, \phi]} e^{i \bar{\psi}}=i_{\sum_{j=0}^{j=3} \frac{1}{j !}[[\phi, \phi], \bar{\psi}]^{\wedge(j)}} ;
$$

(5) $e^{-i \bar{\psi}_{\bar{\psi}}} \mathcal{L}_{\phi} e^{i \bar{\psi}}=\mathcal{L}_{\phi-i} \overline{\bar{\psi}}^{\phi}+i_{\sum_{j=0}^{j=2} \frac{1}{(j+1) !}[[\phi, \bar{\psi}], \bar{\psi}]^{\wedge(j)}}-i_{\sum_{j=0}^{j=2} \frac{1}{(j+2) !}\left[\left[i_{\bar{\psi}} \phi, \bar{\psi}\right], \bar{\psi}\right] \wedge(2)} ;$

(6) $\left.e^{-i_{\bar{\psi}}} e^{-i_{\phi}} \nabla e^{i_{\phi}} e^{i \bar{\psi}}=\nabla-\mathcal{L}_{\bar{\psi}}-\frac{1}{2} i_{[\bar{\psi}, \bar{\psi}]}-\frac{1}{3 !} i_{[[\bar{\psi}, \bar{\psi}], \bar{\psi}]^{\wedge}}-\mathcal{L}_{\phi-i \bar{\psi} \phi}-i_{\sum_{j=0}^{j=2} \frac{1}{(j+1) !}[[\phi, \bar{\psi}], \bar{\psi}]}\right]^{\wedge(j)}$

$$
+i_{\sum_{j=0}^{j=2} \frac{1}{(j+2) !}[[i \bar{\psi} \phi, \bar{\psi}], \bar{\psi}]^{\wedge(2)}}-\frac{1}{2} i_{\sum_{j=0}^{j=3} \frac{1}{j !}[[\phi, \phi], \bar{\psi}]^{\wedge(j)}}-\frac{1}{3 !} i_{\sum_{j=0}^{j=3} \frac{1}{j !}\left[[[\phi, \phi], \phi]^{\wedge}, \bar{\psi}\right]^{\wedge(j)}} .
$$

Proof. All these follows easily from Lemma 3.6.

For (1), note that $\left[\nabla, i_{\phi}\right]=-\mathcal{L}_{\phi}$. By (3.4) and (3.5),

$$
\left[\nabla, i_{\phi}\right]^{(2)}=\left[-\mathcal{L}_{\phi}, i_{\phi}\right]=-i_{[\phi, \phi]} \text { and }\left[\nabla, i_{\phi}\right]^{(3)}=-\left[i_{[\phi, \phi]}, i_{\phi}\right]=-i_{[[\phi, \phi], \phi]^{\wedge}} .
$$

From (2.2) and (2.3) we see that

$$
[[\phi, \phi], \phi]^{\wedge} \in A^{0,2}\left(M, T^{1,0}\right) \text { and }\left[[[\phi, \phi], \phi]^{\wedge}, \phi\right]^{\wedge}=0,
$$

so

$$
\left.\left[\nabla, i_{\phi}\right]^{(4)}=-i_{[[\phi, \phi], \phi]^{\wedge(2)}}=-i_{\left[[[\phi, \phi], \phi]^{\wedge}, \phi\right.}\right]^{\wedge}=0 .
$$

For (2), by Lemma 3.7, we have

$$
\left[\nabla^{1,0}, i_{\phi}\right]=-\mathcal{L}_{\phi}^{1,0},\left[\nabla^{1,0}, i_{\phi}\right]^{(2)}=\left[-\mathcal{L}_{\phi}^{1,0}, i_{\phi}\right]=-i_{[\phi, \phi]}{ }^{A^{0,2}\left(T^{1,0}\right)},\left[\nabla^{1,0}, i_{\phi}\right]^{(3)}=0
$$

and

$$
\left[\nabla^{0,1}, i_{\phi}\right]=-\mathcal{L}_{\phi}^{0,1},\left[\nabla^{0,1}, i_{\phi}\right]^{(2)}=\left[-\mathcal{L}_{\phi}^{0,1}, i_{\phi}\right]=0 .
$$

For (3), we note that

$$
\begin{aligned}
{\left[i_{\theta}, i_{\phi}\right] } & =i_{[\theta, \phi]}, \text { where }[\theta, \phi]^{\wedge} \in A^{1,1}\left(M, T^{0,1}\right) \oplus A^{2,0}\left(M, T^{1,0}\right), \\
{\left[i_{\theta}, i_{\phi}\right]^{(2)} } & =i_{[\theta, \phi]^{\wedge(2)}}, \text { where }[\theta, \phi]^{\wedge(2)} \in A^{0,2}\left(M, T^{0,1}\right) \oplus A^{1,1}\left(M, T^{1,0}\right), \\
{\left[i_{\theta}, i_{\phi}\right]^{(3)} } & =i_{[\theta, \phi]^{\wedge(3)}}, \text { where }[\theta, \phi]^{\wedge(3)} \in A^{0,2}\left(M, T^{1,0}\right),
\end{aligned}
$$

and so that $\left[i_{\theta}, i_{\phi}\right]^{(4)}=i_{[\theta, \phi]^{\wedge(4)}}=0$. Also, $\left[i_{\bar{\theta}}, i_{\phi}\right]=i_{[\bar{\theta}, \phi]^{\wedge}}=0$. 
For (4), we note that $\left[i_{\phi}, i_{\bar{\psi}}\right]^{(2)}=i_{[\phi, \bar{\psi}]^{\wedge(2)}}$ and $[\phi, \bar{\psi}]^{\wedge(2)} \in A^{1,0}\left(M, T^{0,1}\right)$ implies that $\left[i_{\phi}, i_{\bar{\psi}}\right]^{(3)}=\left[\left[i_{\phi}, i_{\bar{\psi}}\right]^{(2)}, i_{\bar{\psi}}\right]=\left[i_{[\phi, \bar{\psi}]^{\wedge(2)}}, i_{\bar{\psi}}\right]=0$. Similarly,

$$
\left[[\phi, \phi]^{A^{0,2}\left(T^{1,0}\right)}, \bar{\psi}\right]^{\wedge(4)}=\left[[\phi, \phi]^{A^{1,1}\left(T^{1,0}\right)}, \bar{\psi}\right]^{\wedge(3)}=\left[[\phi, \phi]^{A^{0,2}\left(T^{0,1}\right)}, \bar{\psi}\right]^{\wedge(3)}=0,
$$

which implies that $\left[i_{[\phi, \phi]}, i_{\bar{\psi}}\right]^{(4)}=0$.

For (5), first by $(2.4)$ we have $\left[\mathcal{L}_{\phi}, i_{\bar{\psi}}\right]=i_{[\phi, \bar{\psi}]}-\mathcal{L}_{i_{\bar{\psi}} \phi}$. And so

$$
\left[\mathcal{L}_{\phi}, i_{\bar{\psi}}\right]^{(2)}=\left[i_{[\phi, \bar{\psi}]}-\mathcal{L}_{i_{\bar{\psi}} \phi}, i_{\bar{\psi}}\right]=i_{[[\phi, \bar{\psi}], \bar{\psi}]^{\wedge}}-i_{\left[i_{\bar{\psi}} \phi, \bar{\psi}\right]},
$$

where we have used the fact that $\mathcal{L}_{i_{\bar{\psi}} i_{\bar{\psi}} \phi}=0$ since $i_{\bar{\psi}} i_{\bar{\psi}} \phi=0$. Similar to (2.3), by $[$ KMS93, pp. 70, Thm $8.7,(6)]$ we have

$$
[\phi, \bar{\psi}] \in A^{1,1}\left(M, T^{1,0}\right) \oplus A^{1,1}\left(M, T^{0,1}\right) \oplus A^{0,2}\left(M, T^{0,1}\right) \oplus A^{2,0}\left(M, T^{1,0}\right),
$$

and

$$
\begin{aligned}
& {\left[[\phi, \bar{\psi}]^{A^{1,1}\left(T^{1,0}\right)}, \bar{\psi}\right]^{\wedge(3)}=\left[[\phi, \bar{\psi}]^{A^{1,1}\left(T^{0,1}\right)}, \bar{\psi}\right]^{\wedge(2)}=0,} \\
& {\left[[\phi, \bar{\psi}]^{A^{0,2}\left(T^{0,1}\right)}, \bar{\psi}\right]^{\wedge(3)}=\left[[\phi, \bar{\psi}]^{A^{2,0}\left(T^{1,0}\right)}, \bar{\psi}\right]^{\wedge(2)}=0,}
\end{aligned}
$$

which implies that $[[\phi, \bar{\psi}], \bar{\psi}]^{\wedge(3)}=0$. Similarly, $\left[\left[i_{\bar{\psi}} \phi, \bar{\psi}\right], \bar{\psi}\right]^{\wedge(3)}=0$. Hence

$$
\begin{aligned}
& {\left[\mathcal{L}_{\phi}, i_{\bar{\psi}}\right]^{(3)}=i_{\left[[[\phi, \bar{\psi}] \bar{\psi}]^{\wedge}-\left[i_{\bar{\psi}} \phi, \bar{\psi}\right], \bar{\psi}\right]^{\wedge}}=i_{[[\phi, \bar{\psi}] \bar{\psi}]^{\wedge(2)}-\left[\left[i_{\bar{\psi}} \phi, \bar{\psi}\right], \bar{\psi}\right]^{\wedge}}} \\
& \left.\left[\mathcal{L}_{\phi}, i_{\bar{\psi}}\right]^{(4)}=i_{[[\phi, \bar{\psi}]} \bar{\psi}\right]^{\wedge(3)}-\left[\left[i_{\bar{\psi}} \phi, \bar{\psi}\right], \bar{\psi}\right]^{\wedge(2)}=-i_{\left[\left[i_{\bar{\psi}} \phi, \bar{\psi}\right], \bar{\psi}\right]^{\wedge(2)}} \\
& {\left[\mathcal{L}_{\phi}, i_{\bar{\psi}}\right]^{(5)}=-i_{\left[\left[i_{\bar{\psi}} \phi, \bar{\psi}\right], \bar{\psi}\right]^{\wedge(3)}}=0 .}
\end{aligned}
$$

For (6), first we have

$$
\begin{aligned}
& e^{-i_{\bar{\psi}}} e^{-i_{\phi}} \nabla e^{i_{\phi}} e^{i_{\bar{\psi}}} \\
= & e^{-i \bar{\psi}}\left(\nabla-\mathcal{L}_{\phi}-\frac{1}{2} i_{[\phi, \phi]}-\frac{1}{3 !} i_{[[\phi, \phi], \phi]^{\wedge}}\right) e^{i \bar{\psi}} \\
= & \nabla-\mathcal{L}_{\bar{\psi}}-\frac{1}{2} i_{[\bar{\psi}, \bar{\psi}]}-\frac{1}{3 !} i_{[[\bar{\psi}, \bar{\psi}], \bar{\psi}]}-e^{-i_{\bar{\psi}}} \mathcal{L}_{\phi} e^{i_{\bar{\psi}}}-\frac{1}{2} e^{-i_{\bar{\psi}}} i_{[\phi, \phi]} e^{i \bar{\psi}}-\frac{1}{3 !} e^{-i_{\bar{\psi}}} i_{[[\phi, \phi], \phi]} e^{i_{\bar{\psi}}},
\end{aligned}
$$

then (6) follows from (4),(5) and the fact that $\left[[[\phi, \phi], \phi]^{\wedge}, \bar{\psi}\right]^{\wedge(4)}=0$.

Remark 3.9. It is not hard to check that the left hand side of these identities are all graded derivations. Hence we can also prove these formulas by using (2.1). In a subsequent paper, we will carry out this approach to prove several extension formulas and study its applications in deformation of complex structures.

Remark 3.10. In the case $E$ is trivial, it's easy to see that $\mathcal{L}_{\phi}^{0,1}=\left[i_{\phi}, \bar{\partial}\right]$ is an algebraic derivation, that is, $\mathcal{L}_{\phi}^{0,1} f=0, \forall f \in A^{0}(M)$. Hence $\mathcal{L}_{\phi}^{0,1}=i_{K}$ for some unique $K \in A^{0,2}\left(M, T^{1,0}\right)$. Let $\left\{e_{i}\right\}$ be a local frame of $T^{1,0}$ and $\left\{\xi^{i}\right\} \subset A^{1,0}(M)$ its dual frame, since $i_{K} \overline{\xi^{i}}=0$ and $i_{K} \xi^{i}=i_{\phi} \bar{\partial} \xi^{i}-\bar{\partial} i_{\phi} \xi^{i}$, we see that $K=K^{i} \otimes e_{i}$, where $K^{i}=i_{\phi} \bar{\partial} \xi^{i}-\bar{\partial} i_{\phi} \xi^{i}$. If furthermore $J$ is integrable, i.e. $M$ is a complex manifold, 
then we may set $e_{i}=\frac{\partial}{\partial z^{i}}$ and $\xi^{i}=d z^{i}$ to be the coordinate frame, it follows that $K=-\bar{\partial} \phi$ and $\mathcal{L}_{\phi}^{0,1}=-i_{\bar{\partial} \phi}$.

Remark 3.11. In the case $E$ is trivial, it's easy to see that $\mathcal{L}_{\phi}^{0,1}=\left[i_{\phi}, \bar{\partial}\right]$ is an algebraic derivation, that is, $\mathcal{L}_{\phi}^{0,1} f=0, \forall f \in A^{0}(M)$. Hence $\mathcal{L}_{\phi}^{0,1}=i_{K}$ for some unique $K \in A^{0,2}\left(M, T^{1,0}\right)$. Let $\left\{e_{i}\right\}$ be a local frame of $T^{1,0}$ and $\left\{\xi^{i}\right\} \subset A^{1,0}(M)$ its dual frame, since $i_{K} \overline{\xi^{i}}=0$ and $i_{K} \xi^{i}=i_{\phi} \bar{\partial} \xi^{i}-\bar{\partial} i_{\phi} \xi^{i}$, we see that $K=K^{i} \otimes e_{i}$, where $K^{i}=i_{\phi} \bar{\partial} \xi^{i}-\bar{\partial} i_{\phi} \xi^{i}$. If furthermore $J$ is integrable, i.e. $M$ is a complex manifold, then we may set $e_{i}=\frac{\partial}{\partial z^{i}}$ and $\xi^{i}=d z^{i}$ to be the coordinate frame, it follows that $K=-\bar{\partial} \phi$ and $\mathcal{L}_{\phi}^{0,1}=-i \bar{\partial} \phi$.

Since $\left(i_{\phi}\right)^{n}=0$, by $(4)$, we see that $\left(i_{\phi+[\phi, \bar{\psi}]^{\wedge}+[\phi, \bar{\psi}]^{\wedge(2)}}\right)^{n}=0$. We failed to give a direct proof of this simple fact. Indeed, we have the following more general result

Proposition 3.12. Let $R$ be an unitary associative algebra over $\mathbb{Q}$ (not necessarily commutative), $x, y \in R$ be nilpotent elements. Assume $x^{N}=0$ for some $N>1$ and $x \in R$ is $k$-commutable with $y$, i.e. $[x, y]^{(k)}=0$, then $\left(\sum_{i=0}^{i=k-1} \frac{1}{i !}[x, y]^{(i)}\right)^{N}=0$ and

$$
e^{-y} e^{x} e^{y}=e^{\sum_{i=0}^{i=k-1} \frac{1}{i !}[x, y]^{(i)}}
$$

Proof. This follows immediately from Lemma 3.6.

Acknowledgements. This note is motivated by the work [LRY15],[RZ17] of LiuRao-Yang and Rao-Zhao on extension formulas. I am grateful to Professor Kefeng Liu for his constant support and encouragement. I would like to thank Kang Wei and Hai-Sheng Liu for useful discussions. I would also like to thank the anonymous referees for their valuable suggestions.

\section{REFERENCES}

[Dem12] J. P. Demailly. Complex analytic and differential geometry. 2012. available at https://wwwfourier.ujf-grenoble.fr/demailly/manuscripts/agbook.pdf.

[FL19] J.-X. Fu and H.-S. Liu. Extension formulae on almost complex manifolds. arXiv:1903.09821, 2019.

[Got05] R. Goto. On deformations of generalized Calabi-Yau, hyperKähler, G2 and Spin(7) structures I. arXiv:math/0512211v1, 2005.

[KMS93] I. Kolář, P. W. Michor, and J. Slovák. Natural operations in differential geometry. SpringerVerlag, Berlin, Heidelberg, New York, 1993.

[LR11] K. Liu and S. Rao. Remarks on the Cartan formula and its applications. Asian Journal of Mathematics, 16(1):157-169, 2011.

[LRY15] K. Liu, S. Rao, and X. Yang. Quasi-isometry and deformations of Calabi-Yau manifolds. Inventiones mathematicae, 199(2):423-453, 2015.

[Man05] M. Manetti. Lectures on deformations of complex manifolds. arXiv:math/0507286v1 [math.AG], 2005.

[Mic86] P. W. Michor. Remarks on the Frölicher-Nijenhuis bracket. In Proceedings of the Conference on Differential Geometry and its Applications, Brno, volume 1086, pages 197-220, 1986. 
[MK71] J. Morrow and K. Kodaira. Complex manifolds, volume 355. American Mathematical Soc., 1971.

[RZ17] S. Rao and Q. Zhao. Several special complex structures and their deformation properties. The Journal of Geometric Analysis, 2017. https://doi.org/10.1007/s12220-017-9944-7.

[Xia19] W. Xia. Derivations on almost complex manifolds. Proceedings of the American Mathematical Society, 147:559-566, 2019.

Wei Xia, Center of Mathematical Sciences, Zhejiang University, Hangzhou, 310027, CHINA.

E-mail address: xiaweiwei3@126.com 